\title{
Giant Cell Myocarditis with Incessant Ventricular Arrhythmias Treated Successfully with Methylprednisolone and Rat Antithymocyte Globulin
}

\author{
Mudassar Baig and Rob Hatrick \\ Department of Cardiology, Western Sussex Hospitals NHS Trust, Lyndhurst Road, Worthing, West Sussex BN11 2DH, UK \\ Correspondence should be addressed to Mudassar Baig, baigoo2003@yahoo.com
}

Received 20 December 2010; Revised 13 February 2011; Accepted 27 February 2011

Academic Editor: Firat Duru

Copyright ( $) 2011$ M. Baig and R. Hatrick. This is an open access article distributed under the Creative Commons Attribution License, which permits unrestricted use, distribution, and reproduction in any medium, provided the original work is properly cited.

Giant cell myocarditis is an aggressive form of this condition that is typically progressive and unresponsive to usual medical treatment. Here, we describe a 34-year-old patient presenting with incessant ventricular arrhythmias with hemodynamic compromise who required prolonged support in intensive care with an intra-aortic balloon pump (IABP). His Coronary arteries were normal and LV endomyocardial biopsy revealed myocyte necrosis with inflammatory infiltrate of lymphocytes, eosinophils, and giant cells suggestive of giant cell myocarditis. He was successfully treated with pulsed intravenous methylprednisolone and rat antithymocyte globulin (RATG). Despite a good functional cardiac recovery, some months later he developed a fluctuant neck swelling which fine needle aspiration confirmed as tuberculosis.

\section{Introduction}

Giant cell myocarditis (GCM) is a rare and often fatal disorder that used to be a postmortem diagnosis until the last quarter of the 20th century [1]. With the advent of endomyocardial biopsy it is now a more recognized antemortem diagnosis. It generally affects healthy and young individuals with mean age of 42 years (15-60 yrs) with no propensity for gender or ethnicity [1].

Observations in human tissue and data from rat models suggest that giant cell myocarditis is an autoimmune disorder dependent on CD4 lymphocytes. Up to $20 \%$ of GCM cases occur in individuals with other inflammatory or autoimmune disorders, especially inflammatory bowel disease and myasthenia gravis [1].

Common presenting symptoms are congestive cardiac failure $(75 \%)$, ventricular arrhythmias (14\%; incessant forms are less common but typical), heart block (5\%), acute coronary syndrome (6\%), and arterial embolization [1].

Endomyocardial biopsy is usually diagnostic with a sensitivity of $82-85 \%$ [2] and should be considered in all patients with acute heart failure or ventricular arrhythmia who fail to improve despite standard medical care. Despite advances in the understanding of the condition and the trial of several different treatment strategies, mortality from this condition remains high with an overall rate of death or heart transplantation of up to $89 \%$ [3].

\section{Case Report}

We report a case of a 34-year-old Bangladeshi gentleman who presented with chest pain, increasing shortness of breath, and palpitations of 1 week's duration. His past medical history was unremarkable. Physical examination revealed a tachycardia of $155 \mathrm{bpm}$ and $\mathrm{BP}$ of $98 / 73 \mathrm{mmHg}$. Initial routine haematology and chemical pathology results were normal. His admission chest X-ray was also normal. ECG revealed a broad complex tachycardia (QRS duration 128 milliseconds) with a ventricular rate of 150, RBBB morphology, and superior right axis deviation consistent with a fascicular origin (Figure 1).

After initial treatment with IV Amiodarone and Magnesium, he became hypotensive with sustained ventricular 


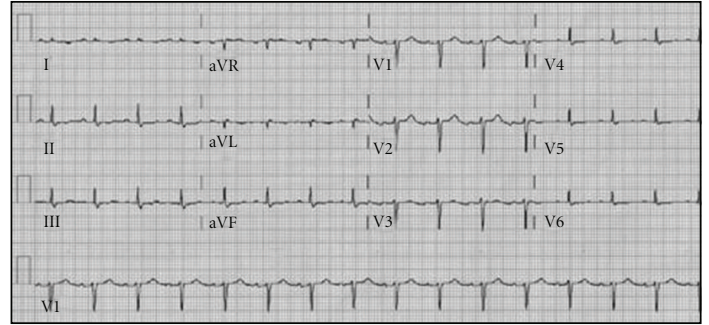

(a)

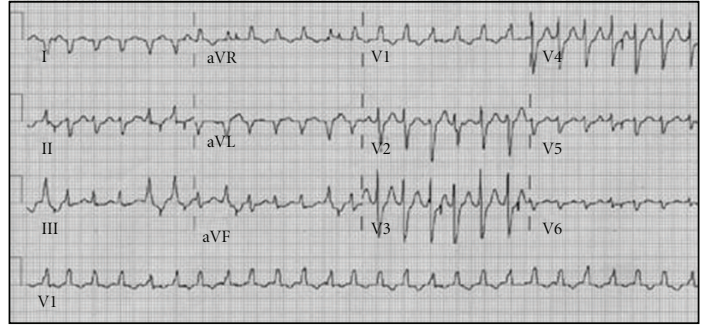

(b)

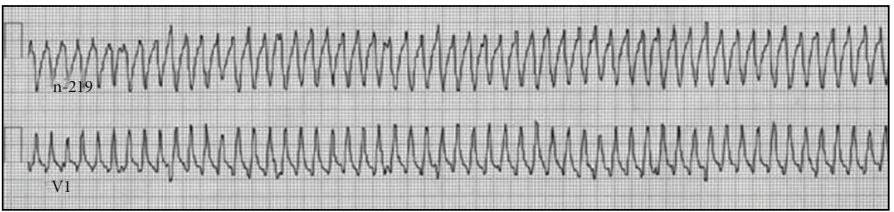

(c)

FIGURE 1: ECGs taken during index admission. Normal sinus rhythm. Sustained monomorphic ventricular tachycardia with right bundle branch block morphology and QRS duration of 130 ms suggesting a fascicular origin. Rhythm strip of faster broad complex tachycardia.

tachycardia; so DC cardioversion was required to restore sinus rhythm. Following this he developed pulmonary oedema and because of hemodynamic compromise he was intubated and transferred to intensive care. He was loaded with amiodarone and given inotropic support but remained unstable with ongoing episodes of sustained VT. This was predominantly fascicular VT with occasional broader complex VT with left bundle branch (LBBB) morphology. Multiple cardioversions were required.

His echocardiogram showed a dilated left ventricle with global hypokinesia and an estimated ejection fraction (EF) of $25 \%$ using Simpson's rule. There was moderate functional mitral regurgitation and elevated estimated pulmonary artery pressure.

On day 5 of his admission he became septic with a temperature of $38^{\circ} \mathrm{C}$, C-reactive protein (CRP) of $120 \mathrm{mg} / \mathrm{dL}$, and white cell count (WCC) of $12 \times 10^{9} / \mathrm{L}$. He developed renal (urea $20.3 \mathrm{mg} / \mathrm{dL}$, creatinine $153 \mathrm{mmol} / \mathrm{lit}$ ) and hepatic (bilirubin $20 \mathrm{mg} / \mathrm{dL}$, ALP $148 \mathrm{IU} / \mathrm{lit}$, ALT $2511 \mathrm{IU} / \mathrm{lit}$, and AST 4187 IU/lit) impairment. He continued to have episodes of sustained VT with hypotension and oliguria, and so an intra-aortic balloon pump (IABP) was inserted and a Lidocaine infusion commenced. He was started on antibiotics (Vancomycin and Levofloxacin). His condition improved initially with improvement of renal and hepatic function but he remained unstable with ongoing episodes of fascicular VT requiring repeated cardioversion. He was eventually transferred to a transplant centre where he had a coronary angiogram (unobstructed coronary arteries) and a blind LV endomyocardial biopsy. The biopsy revealed myocyte necrosis with an inflammatory infiltrate of lymphocytes, eosinophils, and giant cells suggestive of giant cell myocarditis (Figures 2(a) and 2(b)). He was given 3 doses of pulsed IV methylprednisolone and rat antithymocyte globulin (RATG) with dramatic cessation of his incessant VT followed by a sustained hemodynamic improvement. He was commenced on conventional heart failure medication including an ACEinhibitor (Lisinopril), a beta-blocker (Carvedilol), and an aldosterone antagonist (Spironolactone) and hence some of the functional recovery may have been due to guidelinebased care as well as to immunosuppression.

$\mathrm{He}$ was readmitted after 6 months with a fluctuant swelling in the right anterior triangle of the neck consistent with a lymph node. Fine needle aspiration demonstrated Acid Fast Bacilli, and a diagnosis of lymph node tuberculosis was made. He was treated with a 6-month course consisting of standard quadruple therapy for 2 months, followed by 4 months of dual antitubercular therapy. It is difficult to know whether he contracted or reactivated tuberculosis as a result of his immunosuppression, or whether the GCM was complicating preexisting tuberculosis.

Immunosuppression with Prednisolone has continued for a long term although the dose has slowly been reduced to $5 \mathrm{mg}$ bd. A cardiac MRI at 18-month followup demonstrated patchy areas of subendocardial enhancement in the left ventricle, characteristic of a diagnosis of GCM. At 24-month followup the patient remains clinically well with no limiting breathlessness, chest pain, or presyncope. Echocardiography at 24-month followup showed a dilated LV (end-systolic diameter $5.1 \mathrm{~cm}$, end-diastolic diameter $6.2 \mathrm{~cm}$ ) with moderately impaired LV systolic dysfunction and an ejection fraction of $45 \%$. On cardiopulmonary exercise testing, he managed 15 minutes 49 seconds of the modified Bruce protocol with an $\mathrm{MVO}_{2}$ of $30.1 \mathrm{~mL} / \mathrm{kg} / \mathrm{min}$ and a maximal RER of 1.09. Repeat Holter monitoring throughout his follow-up period demonstrated sinus rhythm with a tendency to sinus bradycardia and short burst of asymptomatic nonsustained VT of up to $9 \mathrm{bts}$. Dizziness correlating with his bradycardic episodes precipitated a need to reduce the Carvedilol dose. In view of this, despite his excellent functional status and improved left ventricular ejection fraction, he has now been listed for an implantable defibrillator (ICD). 


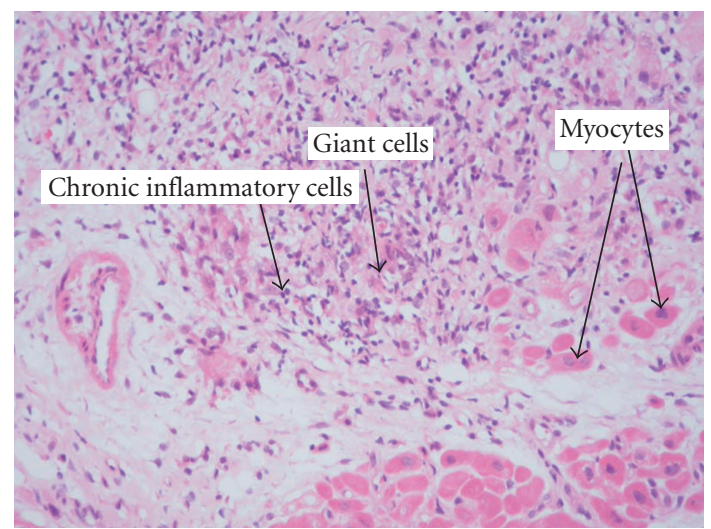

(a)

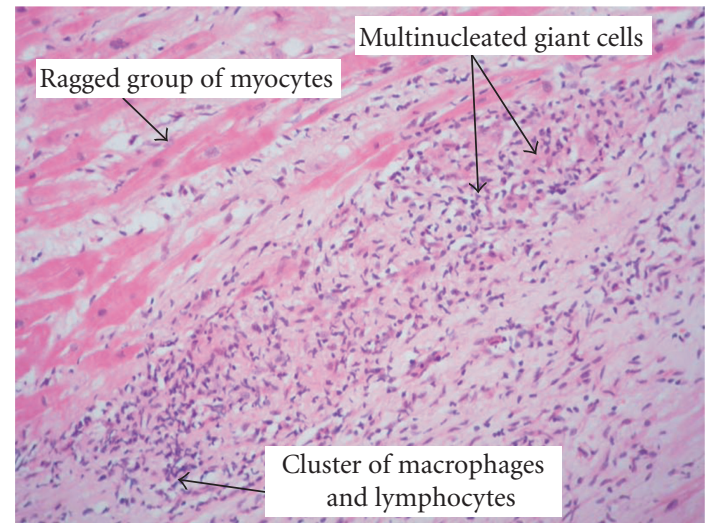

(b)

FIgURE 2: Histology samples from endomyocardial biopsy. Biopsy showing active chronic inflammation with macrophages and giant cells. High-power view of granulomatous focus of myocardial biopsy. Note ill-defined granulomas differentiating it from TB, sarcoidosis, and so forth.

\section{Discussion}

VT is a well-documented clinical presentation of GCM, and it is usually refractory to antiarrhythmic medications as well as DC cardioversion [2]. Ventricular tachycardia in GCM is typically sustained in nature, with moderate QRS width (120-200 ms) and pleomorphic (monomorphic/polymorphic, RBBB/LBBB, and superior/inferior axis) [3]. VT rates are usually relatively slow (average $\sim 155 \mathrm{bpm}$ ) thus not necessarily resulting in hemodynamic compromise.

VT in GCM is due to reentry circuits with myocardial fibrosis and scar tissue providing a substrate for unidirectional block and reentry. A relatively slow heart rate also favours reentry circuits.

No antiarrhythmic medications have been proven to be beneficial in preventing recurrence of VT in GCM, and as disease progresses quickly with worsening malignant arrhythmias and refractory heart failure, it is important that endomyocardial biopsy should be considered early in management of pleomorphic sustained VT with relatively slower heart rate once the more common disorders associated with VT have been excluded. After confirmation of diagnosis, prompt treatment should be considered to prevent further complications.

Definite treatment options for GCM include heart transplantation and immunosuppressive therapy. Heart transplant is supported by a single multicentre study $[1,3-5]$. This study analyzed 63 GCM cases from 36 centres. Only $15 \%$ of 34 patients who underwent heart transplant died within 3 years of transplant despite the recurrence of GCM in grafts, whereas median survival time was only 12.3 months after symptom onset among 22 non-heart-transplant-treated patients given cyclosporine $+/-$ monoclonal antibodies.

Several case reports and the GCM registry suggest that certain combinations of immunosuppressant therapy (but not steroids alone) can prolong transplant-free survival, with the possibility of complete recovery from GCM $[1,3,5]$. In addition to the multicenter GCM registry, the Cleveland Clinic [6] and the university of Ottawa Heart Institute [7] have reported their posttransplantation GCM experiences with similar results. The rate of posttransplantation GCM recurrence (histological) is 20-25\%.

Due to the low incidence of the disease, there is a lack of randomised data to support any specific treatment strategy. However, several immunosuppressants have been used in registry cases with varying success including cyclosporine, azathioprine, steroids, and murine monoclonal Ab (OKT3). In a study by Shioji et al. [8] it was shown that IV Ig was highly effective in ameliorating experimentally induced myocarditis in rats and on this basis RATG has been tried with some success. Our case report adds to the data in support of the use of pulsed methyl-prednisolone and RATG in the treatment of GCM in addition to conventional guideline-based heart failure treatment. This case report also highlights the risk of infection (tuberculosis) as a consequence of lifesaving immunosuppressive therapy. In a study by Cooper et al. [9], a risk of infection up to 1 year following diagnosis was reported in 11 GCM patients treated similarly. There is a lack of evidence addressing the risks and benefit of immunosuppression in this context.

\section{Ethical Approval}

The authors of this paper have certified that they comply with the Principles of Ethical Publishing.

\section{References}

[1] L. T. Cooper, "Giant cell myocarditis: diagnosis and treatment," Herz, vol. 25, no. 3, pp. 291-298, 2000.

[2] R. C. Shields, H. D. Tazelaar, G. J. Berry, and L. T. Cooper, "The role of right ventricular endomyocardial biopsy for idiopathic giant cell myocarditis," Journal of Cardiac Failure, vol. 8, no. 2, pp. 74-78, 2002.

[3] L. T. Cooper Jr., G. J. Berry, and R. Shabetai, "Idiopathic giant cell myocarditis - natural history and treatment. Multicenter giant cell myocarditis study group investigators," England Journal of Medicine, vol. 336, pp. 1860-1866, 1997.

[4] M. Weidenbach, T. Springer, I. Daehnert, K. Klingel, S. Doll, and J. Janoušek, "Giant cell myocarditis mimicking idiopathic fascicular ventricular tachycardia," Journal of Heart and Lung 
Transplantation, vol. 27, no. 2, pp. 238-241, 2008.

[5] H. J. Ankersmit, R. Ullrich, B. Moser et al., "Recovery from giant cell myocarditis with ECMO support and utilisation of polyclonal antithymocyte globulin: a case report," Thoracic and Cardiovascular Surgeon, vol. 54, no. 4, pp. 278-280, 2006.

[6] R. L. Scott, N. B. Ratliff, R. C. Starling, and J. B. Young, "Recurrence of giant cell myocarditis in cardiac allograft," Journal of Heart and Lung Transplantation, vol. 20, no. 3, pp. 375-380, 2001.

[7] R. A. Davies, J. P. Veinot, S. Smith, C. Struthers, P. Hendry, and R. Masters, "Giant cell myocarditis: clinical presentation, bridge to transplantation with mechanical circulatory support, and long-term outcome," Journal of Heart and Lung Transplantation, vol. 21, no. 6, pp. 674-679, 2002.

[8] K. Shioji, C. Kishimoto, Y. Nakayama, and S. Sasayama, "Strain difference in rats with experimental giant cell myocarditis," Japanese Circulation Journal, vol. 64, no. 4, pp. 283-286, 2000.

[9] L. T. Cooper Jr., J. M. Hare, H. D. Tazelaar et al., "Usefulness of immunosuppression for giant cell myocarditis," American Journal of Cardiology, vol. 102, no. 11, pp. 1535-1539, 2008. 


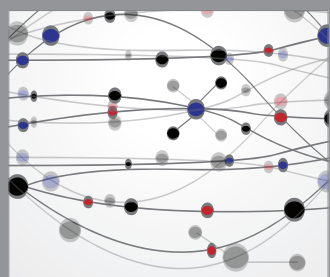

The Scientific World Journal
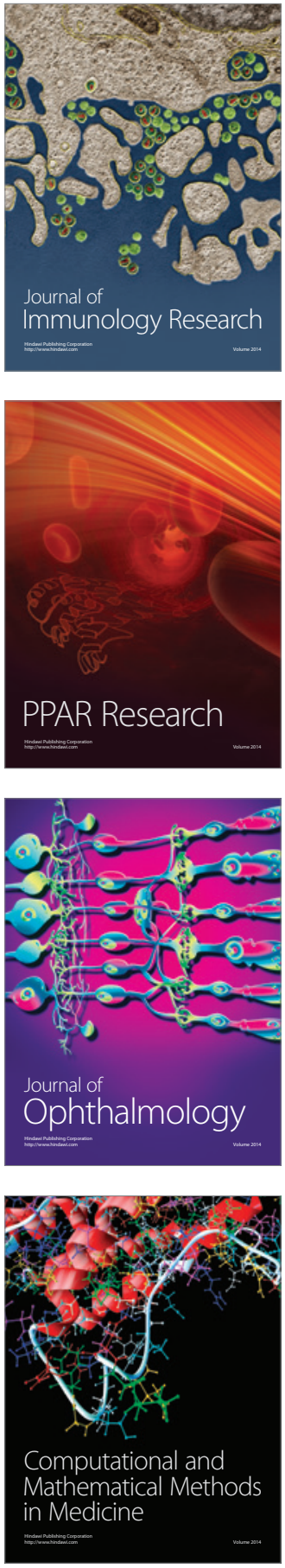

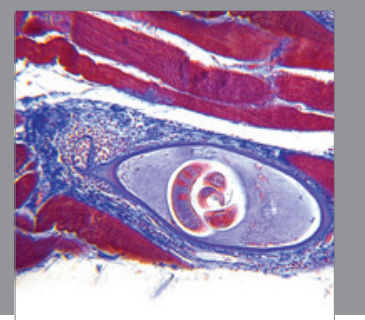

Gastroenterology

Research and Practice
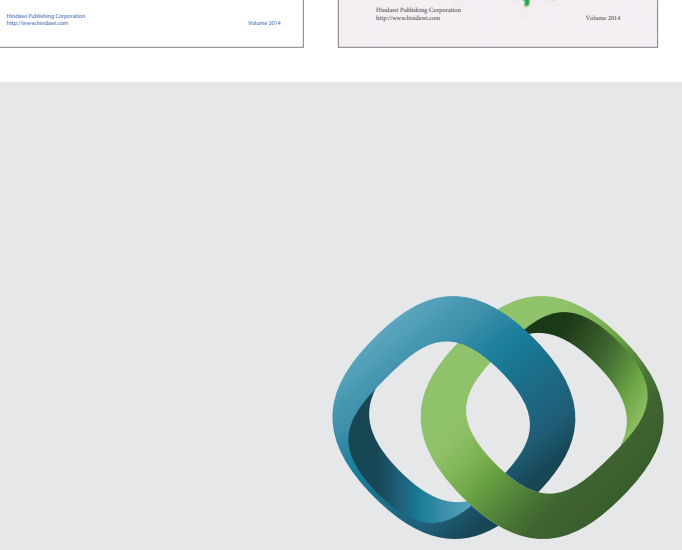

\section{Hindawi}

Submit your manuscripts at

http://www.hindawi.com
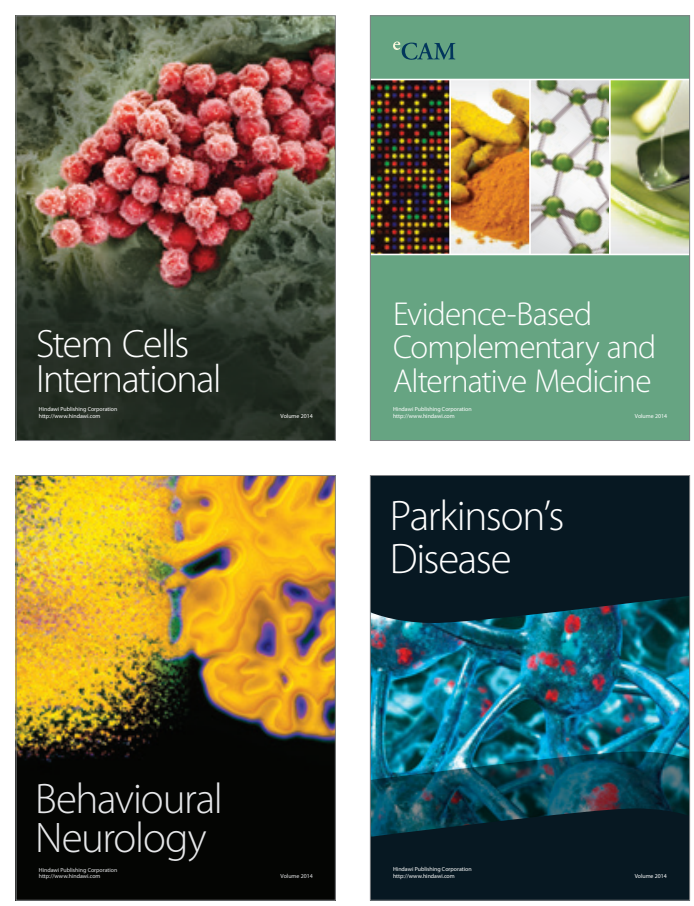

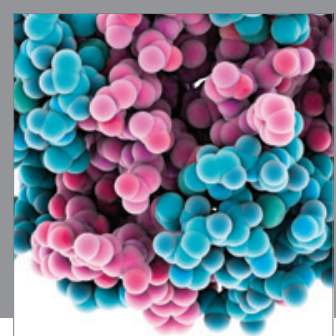

Journal of
Diabetes Research

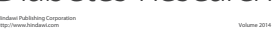

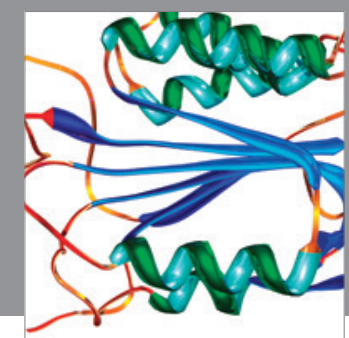

Disease Markers
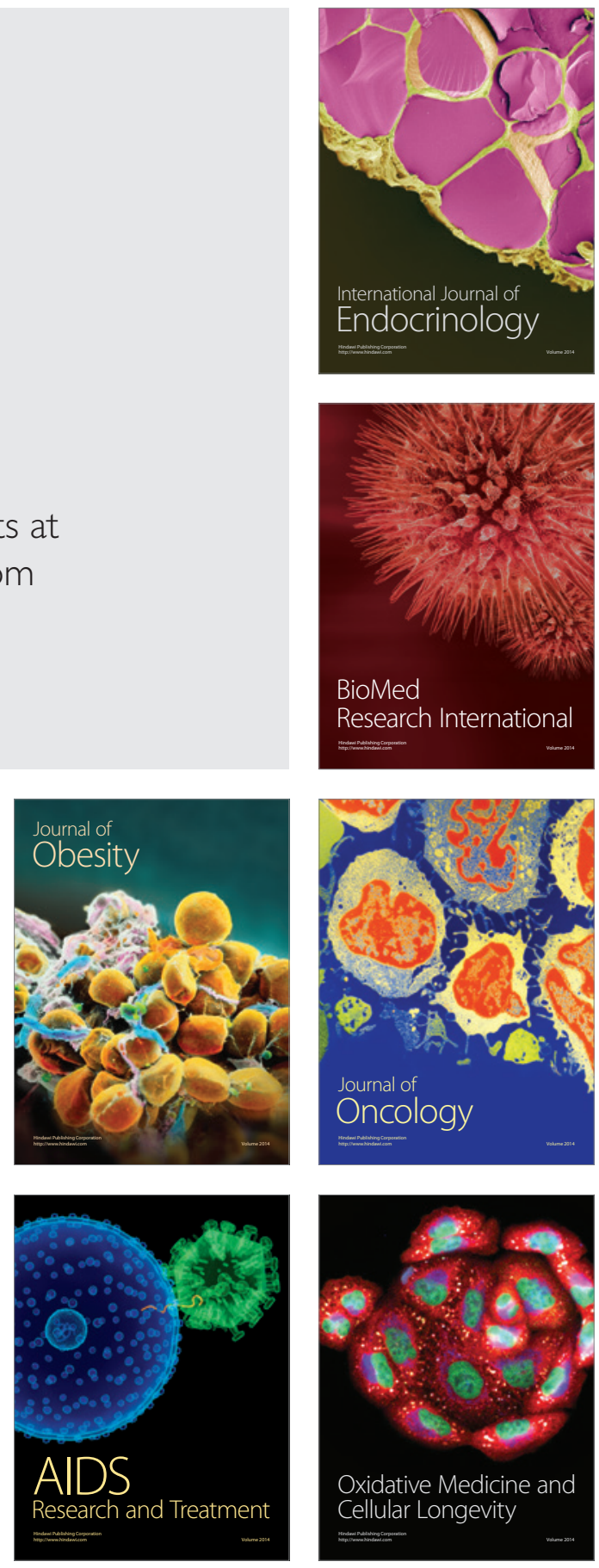